Case Report

\title{
Pantoea agglomerans Infections in Children: Report of Two Cases
}

\author{
Shraddha Siwakoti $\mathbb{D}^{1}{ }^{1}$ Rinku Sah, ${ }^{1}$ Rupa Singh Rajbhandari, ${ }^{2}$ and Basudha Khanal ${ }^{1}$ \\ ${ }^{1}$ Department of Microbiology, B.P. Koirala Institute of Health Sciences, Dharan, Nepal \\ ${ }^{2}$ Department of Pediatrics, B.P. Koirala Institute of Health Sciences, Dharan, Nepal \\ Correspondence should be addressed to Shraddha Siwakoti; shraddha.siwakoti@bpkihs.edu
}

Received 15 September 2017; Revised 16 December 2017; Accepted 3 January 2018; Published 21 January 2018

Academic Editor: Larry A. Rhodes

Copyright (C) 2018 Shraddha Siwakoti et al. This is an open access article distributed under the Creative Commons Attribution License, which permits unrestricted use, distribution, and reproduction in any medium, provided the original work is properly cited.

Introduction. Pantoea agglomerans, primarily an environmental and agricultural organism has been reported as both commensal and pathogen of humans. We present two case reports of $P$. agglomerans infections in children that involved the meninges and bloodstream. Case Presentations. A 6-month-old female baby, diagnosed as congenital hydrocephalus secondary to aqueduct stenosis with ventriculoperitoneal shunt in situ, operated 14 days back was brought to the pediatric emergency with a two-day history of high fever associated with vomiting, irritability, excessive crying, and decreased feeding. Postoperative meningitis was confirmed as cerebrospinal fluid culture revealed $P$. agglomerans. She responded well with a 14-day intravenous (IV) course of ceftriaxone. Also, we report a case of a 3-year-old male child referred to our center with a provisional diagnosis of UTI with chickenpox for further evaluation. During his 24-hour stay at the local hospital, he had received oral antibiotics and urinary catherization. Urine culture of catheter clamp urine was sterile. P. agglomerans was grown in blood culture. He was treated successfully with IV ceftriaxone and amikacin. Conclusion. P. agglomerans can cause postsurgical meningitis and bloodstream infection in children. The clinical course of infection was mild and timely administration of proper antibiotic resulted in a favorable outcome.

\section{Introduction}

Pantoea agglomerans is a gram-negative aerobic bacillus that belongs to the family Enterobacteriaceae. It is primarily an environmental and agricultural organism that inhabits plants, soil, and water. This bacterium has been reported as both commensal and pathogen of animals and humans [1]. Human infections may be associated with trauma caused by penetration of vegetative material and also with secondary bacteremia or nosocomial infections that are related to medical equipment such as intravenous catheters or contaminated intravenous fluids and parenteral nutrition [2-4]. Spontaneously occurring bacteremia and meningitis caused by this pathogen have rarely been reported, especially in children. Here, we present two case reports of $P$. agglomerans infections in children that involved the meninges and bloodstream.

\section{Case Report 1: Meningitis}

This case was a six-month-old female infant, diagnosed as congenital hydrocephalus secondary to aqueduct stenosis with VP shunt in situ. Fourteen days earlier, the child had undergone ventriculoperitoneal (VP) shunt surgery. The patient presented to the pediatric emergency with a two-day history of high fever associated with vomiting (2 episodes), irritability, excessive crying, and decreased feeding. On examination, her heart rate was $136 / \mathrm{min}$, respiratory rate was $60 / \mathrm{min}$, and temperature was $37.7^{\circ} \mathrm{C}$. Central nervous system examination showcased normal cry, hypertonia, decreased power, and exaggerated reflexes on both upper and lower limbs. Pupils were bilaterally equal and reacting to the light. Examination results of the respiratory system, cardiovascular system, and abdomen were within normal limits. The child was treated empirically with IV vancomycin 
and ceftriaxone after collecting the CSF sample by lumbar puncture. CSF analysis showed an elevated white blood cell count of $400 \times 10^{6} / \mathrm{L}$ (95\% polymorphs and 5\% lymphocytes), raised protein level of $141 \mathrm{mg} / \mathrm{dl}$, and decreased glucose level of $40 \mathrm{mg} / \mathrm{dl}$ (blood glucose level: $70 \mathrm{mg} / \mathrm{dl}$ ). Gram stain of direct CSF smear showed few pus cells and few gram negative bacilli. CSF culture on chocolate and 5\% sheep blood agar showed yellow pigmented, smooth surface colonies (Figure 1) and Mac Conkey's agar grew a nonlactose fermenting bacillus after 48-hours of aerobic incubation at $35^{\circ} \mathrm{C}$. Based on biochemical reactions (Table 1), the colony was identified as Pantoea agglomerans. Antimicrobial susceptibility by Kirby-Bauer disc diffusion method showed the isolate to be resistant to ampicillin and sensitive to amikacin, ceftriaxone, ciprofloxacin, cotrimoxazole, and meropenem. After receiving the CSF culture report, vancomycin was discontinued, and IV ceftriaxone was continued for 2 weeks. The child's condition improved, and she was discharged after 15 days of hospital stay.

\section{Case Report 2: Blood Stream Infection}

A 3-year-old male child from the eastern part of Nepal was referred to our tertiary center after 24 hours admission at a local hospital with a provisional diagnosis of UTI with chickenpox for further evaluation. The child had a four-day history of abdominal pain, pain and difficulty with urination, urinary retention, and fever (up to $39^{\circ} \mathrm{C}$ ). Urinary catheterization was done, and oral antibiotic was administered at the local hospital. The patient had also experienced rashes for 3 days, which started from lower limb and was later generalized. The patient had a recent history of UTI one month back. On the current admission, examination revealed temperature to be $37^{\circ} \mathrm{C}$ and skin showed multiple, erythematous rashes with dew drop appearance. Routine urine microscopic examination revealed the presence of 2 to 3 pus cells per high power field. Urine culture of catheter clamp urine did not yield a growth. Abdominal ultrasound showed mild hydronephrosis bilaterally and dense internal echoes within the urinary bladder lumen. A voiding cystourethrogram showed typical findings of a posterior urethral valve (PUV) with grade III vesicoureteral reflux. Blood culture grew Pantoea agglomerans sensitive to ampicillin, amikacin, ceftriaxone, ciprofloxacin, cotrimoxazole, and meropenem. Other laboratory parameters were within normal range. The child was empirically treated with IV ceftriaxone and amikacin which was continued for 7 days after getting the antibiotic sensitivity report. The child was successfully treated and discharged on the 8th day of hospital stay. He is currently being followed up at pediatric outpatient department and managed conservatively for PUV.

\section{Discussion}

Human infections caused by $P$. agglomerans are most often associated with wound infection with plant material or hospital acquired due to contamination of medical equipment and fluids. The most common infections caused by this

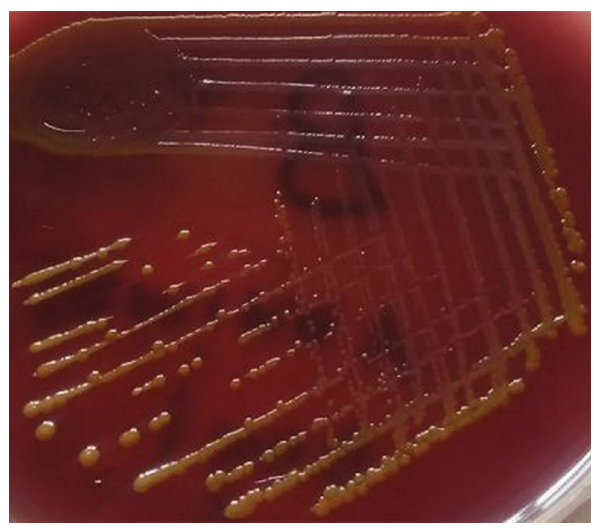

FIgURE 1: Growth of Pantoea agglomerans in blood agar.

Table 1: Biochemical tests to differentiate P. agglomerans.

\begin{tabular}{lc}
\hline Biochemicals & Interpretation \\
\hline Catalase & Produced \\
Oxidase & Not produced \\
Citrate (Simmons) & Utilized \\
Urease, hydrogen sulfide, indole & Not produced \\
Motility & Motile \\
Nitrate & Reduced to nitrite \\
Glucose, xylose, arabinose, maltose, & Fermented \\
trehalose, rhamnose, mannitol & Not fermented \\
Lactose, sucrose, sorbitol & Fermentative \\
Oxidative fermentative (OF) & Positive \\
Methyl red, Voges-Proskauer & Not decarboxylated \\
Lysine, ornithine, arginine &
\end{tabular}

pathogen in children are blood stream infection, abscess, osteomyelitis, septic arthritis, and urinary tract infection. And, the source of these infections as described in literature is due to thorn pricks, infected parenteral fluids, and indwelling catheters [4]. Pantoea spp. seems to be a relatively uncommon cause of meningitis. In our case, the absence of any bacteria other than P. agglomerans on the CSF culture confirmed that this pathogen was responsible for the postoperative meningitis. Similarly, Wang and Fraser have isolated $P$. agglomerans from the brain abscess that developed after infarction and hemicraniectomy [5]. A case report of Pantoea calida causing postsurgical meningitis in a 52-year-old female has been reported by Fritz et al. [6]. Our isolation of $P$. agglomerans from a similar case indicates that Pantoea spp. must be considered as an opportunistic Enterobacteriaceae pathogen responsible for postsurgical meningitis. This $P$. agglomerans isolate displayed in vitro sensitivity to all the commonly used antibiotics, and accordingly, the child recovered with proper antibiotic treatment. A similar finding of antimicrobial sensitivity pattern was reported by Fritz et al. of their P. calida isolate from postsurgical meningitis where the patient had full recovery after 14 days of treatment with meropenem [6]. This depicts that meningitis caused by Pantoea spp. can have 
a mild clinical course, and administration of an effective antibiotic can cure the infection.

In our second case report, $P$. agglomerans was isolated from blood culture from a child. There can be two possible causes for this blood stream infection. It is conceivable that this bacteremia could be secondary to the UTI episode. The child had clinical features of UTI and was diagnosed with PUV, an important risk factor for UTI. Microbiology testing revealed a sterile urine culture which can be explained by the recent antibiotic administration at the local hospital. Instead, this bacteremia could be an event of primary blood stream infection; by definition not secondary to localized foci, as the exact source of bacteremia could not be established. Moreover, there was no identifiable exogenous source of infection. Most of the cases of P. agglomerans bacteremia in the literature have been documented in association with the contamination of intravenous fluid, total parenteral nutrition, and blood products $[2,3]$. Conversely, the spontaneously occurring bacteremia has rarely been reported, especially for children. A study by Cruz et al. reported 23 culture-documented $P$. agglomerans infections in children with 21 central venous line- (CVL-) related bacteremic episodes and 2 nonCVL-associated spontaneous bacteremic episodes over 6 years [4]. Likewise 5 cases of sporadic $P$. agglomerans septicemia in preterm neonates with full recovery of all cases due to proper antibiotic therapy have been reported [7]. A case report of bloodstream infection caused by Pantoea spp. in a vaginally delivered 4-day-old baby from India had a favorable outcome after antibiotic therapy [8]. The outcome in our case was also benign after antibiotic treatment which may be partly due to early diagnosis and the adequacy of the antibiotics. Moreover, we believe that this favorable outcome of our child was probably because of infection caused by relatively less virulent strain. In contrary, there are several previously reported cases in the literature that had a fatal outcome $[3,4,7]$. Bergman et al. reported mortality of 3 cases of sporadic septicemia out of 125 infections with $P$. agglomerans among 6,383 newborns hospitalized in an intensive care unit [9]. This lethal outcome may be due to decline of patient's immunity caused by underlying disease, prematurity, and/or hospital procedures.

To the best of our knowledge, these are the first case reports of $P$. agglomerans causing infections in children from Nepal. Being an uncommon agent of human infection, $P$. agglomerans may be underreported or reported as any other member of Enterobacteriaceae in the routine settings. Due to the ubiquitous presence of $P$. agglomerans in nature, strict compliance to infection control practices would prevent the infection with this agent.

\section{Conclusion}

P. agglomerans can be the opportunistic agent for postsurgical meningitis and pathogen for bloodstream infection in children. The clinical course of infection caused by this bacterium can be mild, probably due to infection caused by relatively less virulent strain and can be treated with proper antibiotic therapy.

\section{Consent}

Written informed consent was obtained from the patient's legal guardian for publication of these case reports.

\section{Conflicts of Interest}

The authors declare that they have no conflicts of interest.

\section{References}

[1] F. Gavini, J. Mergaert, A. Beji et al., "Transfer of Enterobacter agglomerans (Beijerinck 1888) Ewing and Fife 1972 to Pantoea gen. nov. as Pantoea agglomerans comb. nov. and description of Pantoea dispersa sp. nov.," International Journal of Systematic Bacteriology, vol. 39, no. 3, pp. 337-345, 1989.

[2] N. S. Matsaniotis, V. P. Syriopoulou, M. C. Theodoridou, K. G. Tzanetou, and G. I. Mostrou, "Enterobacter sepsis in infants and children due to contaminated intravenous fluids," Infection Control, vol. 5, no. 10, pp. 471-477, 1984.

[3] H. Habsah, M. Zeehaida, H. Van Rostenberghe et al., "An outbreak of Pantoea spp. in a neonatal intensive care unit secondary to contaminated parenteral nutrition," Journal of Hospital Infection, vol. 61, no. 3, pp. 213-218, 2005.

[4] A. T. Cruz, A. C. Cazacu, and C. H. Allen, "Pantoea agglomerans, a plant pathogen causing human disease," Journal of Clinical Microbiology, vol. 45, no. 6, pp. 1989-1992, 2007.

[5] J. Wang and J. F. Fraser, "An intracranial petri dish? formation of abscess in prior large stroke after decompressive hemicraniectomy," World Neurosurgery, vol. 84, no. 5, pp. 1495. e5-1495.e9, 2015.

[6] S. Fritz, N. Cassir, R. Noudel, S. De La Rosa, P.-H. Roche, and M. Drancourtt, "Postsurgical Pantoea calida meningitis: a case report," Journal of Medical Case Reports, vol. 8, no. 1, p. 195, 2014.

[7] N. Y. Aly, H. N. Salmeen, R. A. Lila, and P. A. Nagaraja, "Pantoea agglomerans bloodstream infection in preterm neonates," Medical Principles and Practice, vol. 17, no. 6, pp. 500-503, 2008.

[8] S. Tiwari and S. S. Beriha, "Pantoea species causing early onset neonatal sepsis: a case report," Journal of Medical Case Reports, vol. 9, no. 1, p. 188, 2015.

[9] K. A. Bergman, J. P. Arends, and E. H. Schölvinck, "Pantoea agglomerans septicemia in three newborn infants," Pediatric Infectious Disease Journal, vol. 26, no. 5, pp. 453-454, 2007. 


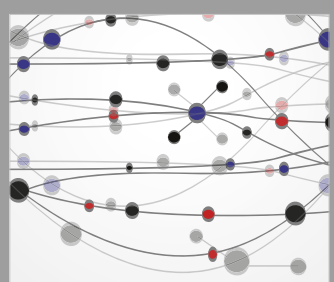

The Scientific World Journal
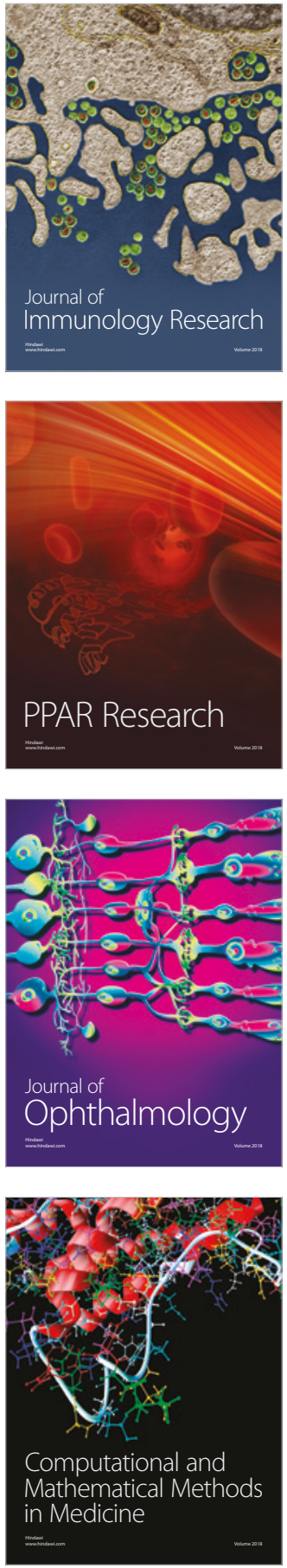

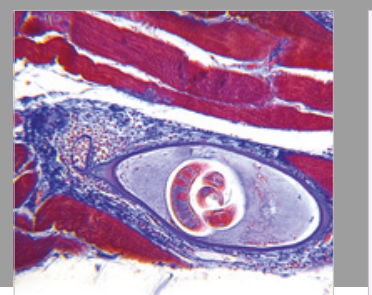

Gastroenterology Research and Practice

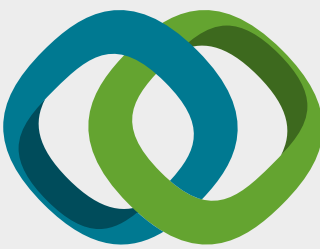

\section{Hindawi}

Submit your manuscripts at

www.hindawi.com
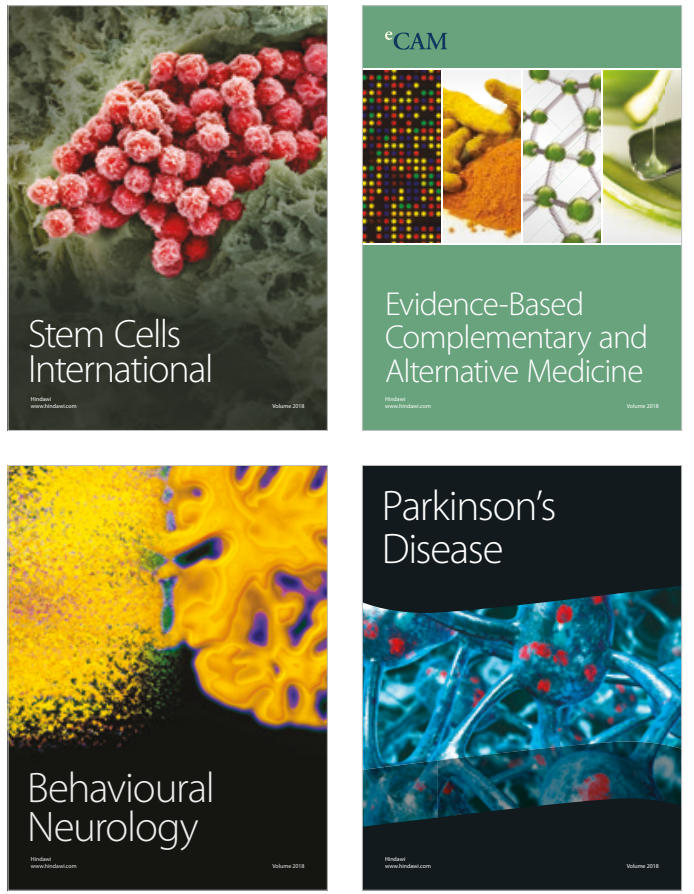

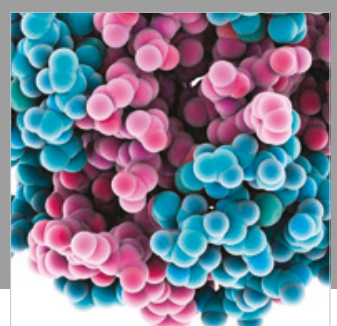

ournal of

Diabetes Research

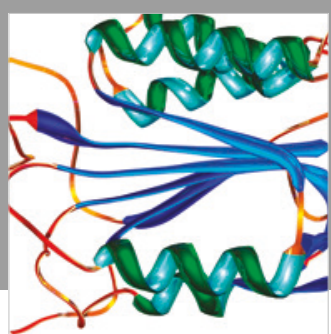

Disease Markers
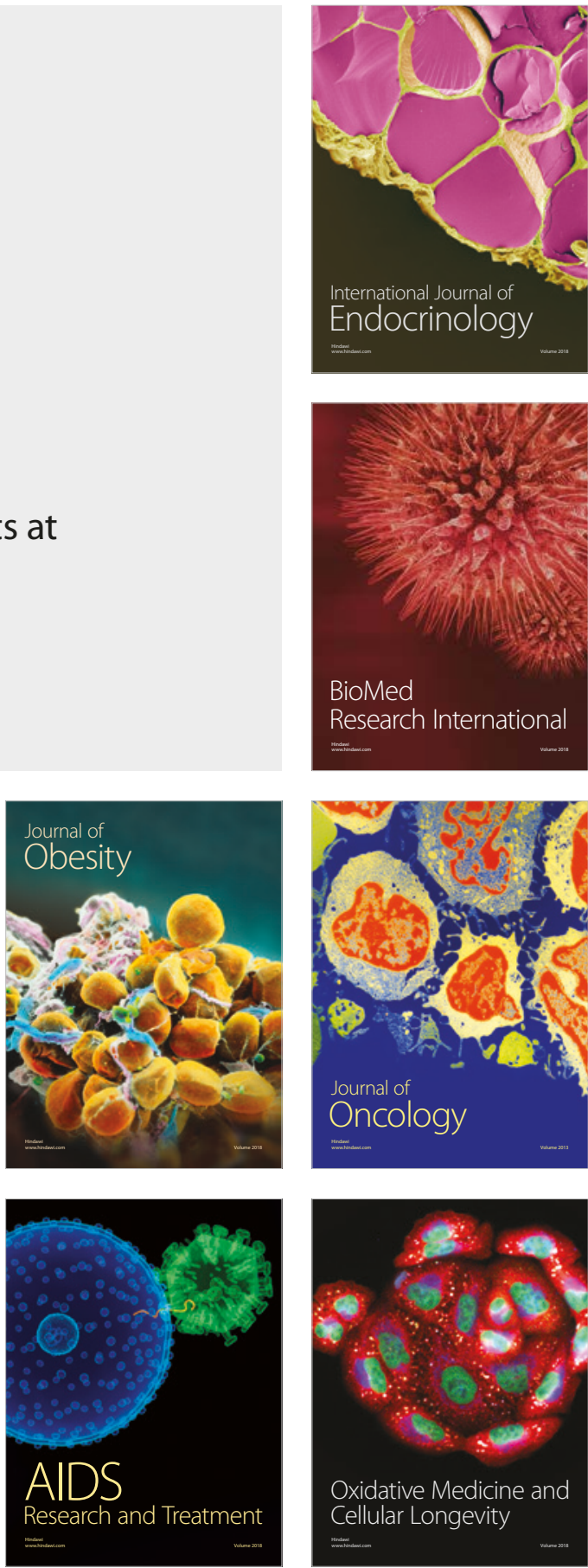